\title{
Index autorum ad Vol. 169
}

Confecit: C. Loeb-Schürch

$(\mathrm{V})=$ Report - Vortrag - Communication $(\mathrm{B})=$ Book Reviews - Buchbesprechungen - Livres nouveaux

Aspinall, P. A. 299 Aulhorn, E. 50 (V)

Frey, R. 397 (B) Frezzotti, R. 321

Bellone, G. 290 Bessière, E. 397 (B) Bialas, B. 371 Binkhorst, P. G. 401 Boba, A. 397 (B)

Bolmers, D. J. M. 241 Brunette, J.-R. 141 (V)

Cambie, E. 255, 362

Gabreëls, F. J. M. 241 Gabreëls-Festen, A. 241 Gallenga, P. E. 290 Gierek, A. 371 Glaser, J. S. 397 (B) Gligo, D. 38 (V) Graham, M. D. 320 (B) Grosz, I. von 320 (B) Guerra, R. 321

Daicker, B. 377 De Laey, J. J., v. Laey, J. J. de De Rouck, A., v. Rouck, A. de Dreyer, V. 61 (V) Dubois-Poulsen, A. 13 (V) Dwyer-Joyce, P. 1 (V)

Eisner, G. 399 (B) El-Shewy, T. M. 285 Enoksson, P. 176 (V) Erbakan, S. 99 (V) Estévez, O. 70 (V)

Faggioni, R. 333, 411 Follmann, P. 192 (V) Fox, S. L. 399 (B) Francois, J. 234 (V), 255, 345, 362,452

Hamburg, A. 401 Hammami, H. 333 Harms, H. 50 (V) Henkes, H. E. 151 (V) Hinzpeter, E. N. 390 Hollwich, F. 398 (B) Hruby, K. 23 (V) Huber, A. Ill (V)

Joosten, E. M. G. 241

Kern, F. 397 (B) Köhler, U. 432 Kolder, H. E. 127 (V)

Laey, J. J. de 255, 362 Lawton Smith, J. 397 (B) Leopold, I. H. 398 (B)

476 Index

Leydhecker, W. 399 (B) Lith, G. H. M. van 151 (V) Luttenberger, I. 38 (V)

Mayrhofer, O. 397 (B) Monnier, M. 160 (V) Miiller, K.-M. 311 Müller, M. 432

Naumann, G. 390 Nordmann, J. 459

Ortbauer, R. 390 Ourgaud, A. G. 203 (V)

Pasquarelli, A. 290 Peleska, M. 184 (V) Pinckers, A. J. L. G. 241

Riede, U. N. 377 Rouck, A. de 255

Saari, M. 326 Scheffer, C. H. 401 Schwarz, E. Ch. 442 Scouras, J. 411

ad Vol. 169

Shikano, S.-I. 400 (B) Sosnierz, M. 371 Streiff, E. B. 333, 411 Szymański, A. 371

Terragna, A. 321

Theodossiadis, G. 416

Tommila, V. $90(\mathrm{~V})$

Tongos, D. 416

Tosi, P. 321

Tweel, L. H. van der 70 (V) 
Van der Tweel, L. H., v. Tweel, L. H.

van der Van Lith, G. H. M., v. Lith, G. H. M.

van Verriest, G. 82 (V), 255 Victoria-Troncoso, V. 452 Vogel, M. H. 311 Von Grosz, I., v.

Grosz, I. von

Witting, C. 311

Y. Yamada, E. 400 (B)

Zellweger, J. P. 333 